OPEN ACCESS

Edited by:

Annalisa Trama,

Istituto Nazionale dei Tumori (IRCCS),

Reviewed by:

Ka chun Cheung,

University of Adelaide, Australia

Xiaoxia $\mathrm{Hu}$,

Ruijin Hospital, China

*Correspondence:

Xueyi Pan

xypan88@163.com

${ }^{\dagger}$ These authors have contributed equally to this work and

share first authorship

Specialty section: This article was submitted to Hematologic Malignancies, a section of the journal

Frontiers in Oncology

Received: 28 April 2021

Accepted: 31 July 2021

Published: 18 August 2021

Citation:

Zhu Q, Yang S, Zeng W, Li M, Guan Z, Zhou L, Wang H, Liu Y,

Gao Y, Qiu S, Chen C, Li H,

Zheng S, Yuan $Y$, Zhang $H$ and Pan $X$ (2021) A Real-World

Observation of Eltrombopag and Recombinant Human Thrombopoietin (rhTPO) in

Lymphoma Patients With

Chemotherapy Induced

Thrombocytopenia.

Front. Oncol. 11:701539.

doi: 10.3389/fonc.2021.701539

\section{A Real-World Observation of Eltrombopag and Recombinant Human Thrombopoietin (rhTPO) in Lymphoma Patients With Chemotherapy Induced Thrombocytopenia}

\author{
Qiuhua Zhu ${ }^{\dagger}$, Shimei Yang ${ }^{\dagger}$, Wenbin Zeng, Mingjie Li, Zebing Guan, Lanlan Zhou, \\ Hong Wang, Yanan Liu, Yanmin Gao, Shiqiu Qiu, Chaolun Chen, Huan Li, \\ Shanshan Zheng, Yuemei Yuan, Hanling Zhang and Xueyi Pan* \\ Department of Hematology, The First Affiliated Hospital of Guangdong Pharmaceutical University, Guangdong, China
}

This real-world, observational study aimed to assess and compare the clinical efficacy and safety of eltrombopag with recombinant human thrombopoietin (rhTPO) in the treatment of chemotherapy induced thrombocytopenia (CIT) in patients with lymphoma. One hundred and fifty-three patients who experienced grade 3 or 4 thrombocytopenia after chemotherapy for lymphoma were enrolled, 51 of which were treated with eltrombopag, 50 with rhTPO, and 52 patients with no drug treatment were served as the control group. The lowest platelet level and mean platelet counts at Day 5, Day 7, and Day 10 were significantly higher in both the eltrombopag group $(P=.041, .003, .000, .000)$ and rhTPO group $(P=.005, .005, .000, .000)$ than the control, but there was no difference between treatment with eltrombopag and rhTPO. Similarly, days required for the recovery of platelet counts to $\geq 50 \times 10^{9} / \mathrm{L}$ and $\geq 75 \times 10^{9} / \mathrm{L}$ were not different between the two treatment groups but significantly higher than the control group $(P<.05)$. Rates of bleeding and platelet transfusion were all significantly reduced in patients treated with eltrombopag $(P=.031, .032)$ or rhTPO $(P=.017, .009)$ when compared to the control. Treatment-related adverse events (AEs) were reported in 7 (13.7\%) and 6 (12.0\%) patients in the eltrombopag and rhTPO groups, respectively, all being mild and transient in nature. In conclusion, both eltrombopag and rhTPO were effective and safe in the treatment of thrombocytopenia after chemotherapy for lymphoma.

Keywords: eltrombopag, recombinant human thrombopoietin (rhTPO), lymphoma, chemotherapy, thrombocytopaenia 


\section{INTRODUCTION}

Lymphoma is a malignant tumor originating from lymph nodes and (or) extranodal lymphoid tissues. The main symptoms include painless lymphadenopathy, hepatosplenomegaly, accompanying whole-body and multi-organ reactions such as fever, drenching night sweat, wasting, and itching (1). Myelosuppression, presented as different degrees of leukopenia, thrombocytopenia and anemia, is prevalent in patients with lymphoma who are undergoing chemotherapy (2). Chemotherapy-induced thrombocytopenia (CIT) not only increases the risk of hemorrhage, but also causes chemotherapy dose delays, dose reductions, or even treatment discontinuation that may result in prolonged hospitalization, increased medical costs, and reduced progression-free and overall survival (3-5).

At present, the treatment of CIT for lymphoma is very limited, which mainly include platelet transfusion and recombinant human interleukin-11 (rhIL-11). Platelet transfusion is the fastest and most effective treatment for severe CIT; however, as a rescue therapy, it is associated with temporary effectiveness, adverse reactions, and loss of response with repeated administrations $(3,6)$. rhIL-11 is the only platelet growth factor approved by the United States (US) Food and Drug Administration (FDA) and China National Medical Products Administration (NMPA) for CIT, but its use is severely restricted by the narrow therapeutic index and significant side effects including edema and arrhythmia (7). Therefore, novel treatment options are needed to improve the efficacy and safety outcomes of thrombocytopenia after chemotherapy for lymphoma.

Thrombopoietic agents were designed to stimulate the c-mpl receptor that leads to megakaryocyte maturation and platelet production (7). The rhTPO is a full-length glycosylated thrombopoietin prepared from Chinese hamster by recombinant DNA technology, which has a pharmacological effect similar to endogenous thrombopoietin (TPO) (8). It is the only thrombopoietin receptor agonist (TPO-RA) that receives market approval in China for the treatment of CIT (9, 10). Eltrombopag, as a chemically synthesized, orally available, small molecule, nonpeptide TPO-RA, has been approved for the treatment of chronic immune thrombocytopenia, hepatitis $\mathrm{C}$ virusrelated thrombocytopenia, and (refractory) severe aplastic anemia. Recent studies showed that eltrombopag played an antiproliferative effect in hematologic malignancies, raising the possibility of its use in CIT treatment (11).

It was reported in several randomized, placebo-controlled phase I and II clinical trials that eltrombopag has achieved objective curative effect on thrombocytopenia during chemotherapy cycles, especially in advanced solid tumor patients receiving gemcitabine -based chemotherapy and patients with acute leukemia $(6,12-14)$. Currently, there's no study that assessed TPO-RAs for treatment of CIT in patients with lymphoma, and it is unknown whether the two TPO-RAs, rhTPO and eltrombopag, are comparable with regard to the clinical effectiveness and tolerability in CIT treatment. Using the data from a tertiary clinical practice in China, this large sample, observational study innovatively reviewed and compared the efficacy and safety profiles of eltrombopag to the marketapproved rhTPO in the treatment of thrombocytopenia after chemotherapy for lymphoma.

\section{MATERIALS AND METHODS}

This retrospective study was approved by the Ethics Committee of the First Affiliated Hospital of Guangdong Pharmaceutical University. Between April 2017 and September 2020, patients with histopathologically confirmed lymphoma who also experienced grade 3 or 4 thrombocytopenia (platelet counts $<30 \times 10^{9} / \mathrm{L}$ ) after chemotherapy (15) at the Hematology Department of our institution were enrolled. For the same patient who met the above criteria in more than one chemotherapy cycles, data of the earliest occurrence of PLT $<30 \times 10^{9} / \mathrm{L}$ that were treated either with eltrombopag, rhTPO, or no drug treatment were included in this study. Exclusion criteria included tumors secondary to lymphoma and concomitant hemophagocytic syndrome. Patients with documented treatment history of eltrombopag or rhTPO after chemotherapy for lymphoma in other hospitals, concurrent treatment with both eltrombopag and rhTPO, eltrombopag and rhIL-11, or rhTPO and rhIL-11, were also excluded. The need for informed consent was waived by the committee.

One hundred and fifty-three lymphoma patients (90 males, 63 females) with CIT were consecutively enrolled in this study. Among them, 51 patients were treated with eltrombopag, 50 patients were treated with rhTPO, and 52 patients without drug treatment were selected as controls. Eltrombopag or rhTPO was initiated when platelet counts fell below $30 \times 10^{9} / \mathrm{L}$ after chemotherapy. The starting dose of eltrombopag was $50 \mathrm{mg}$ daily. The dose was adjusted to 75 $\mathrm{mg} /$ day when the platelet counts decreased to less than $10 \times 10^{9} / \mathrm{L}$. For patients who received rhTPO, daily injection of $15,000 \mathrm{U}$ was given subcutaneously. In all patients, platelet transfusion was initiated when the platelet level was less than $20 \times 10^{9} / \mathrm{L}$. If platelets increased to more than $100 \times 10^{9} / \mathrm{L}$, or $50 \times 10^{9} / \mathrm{L}$ more than the baseline, treatment of eltrombopag or rhTPO was stopped.

Demographic, socioeconomic, and clinical characteristics were obtained from patients. Efficacy variables, including platelet counts at baseline and on day 3, 5, 7, 10, and the lowest platelet counts after treatment, days required for the recovery of platelet counts to $\geq 50 \times 10^{9} / \mathrm{L}$ and $\geq 75 \times 10^{9} / \mathrm{L}$, respectively, duration of platelet counts $<50 \times 10^{9} / \mathrm{L}$, platelet transfusion frequency and volume, and the modified World Health Organization (WHO) bleeding grades (10), were assessed. Adverse events (AEs) were monitored and assessed according to the Common Terminology Criteria for Adverse Events (CTCAE) Version 5.0 (15). Increased transaminases were defined as alanine aminotransferase (ALT) or aspartate aminotransferase (AST) $\geq 3 \times$ the upper limit of normal (ULN), and hyperbilirubinemia as total bilirubin $\geq 1.5 \times \mathrm{ULN}$.

\section{Statistical Analysis}

All statistical analyses were performed using SPSS 19.0 (IBM Corp., Armonk, NY). Continuous variables were summarized by mean, standard deviation (SD), median, and range, while categorical variables by number and frequencies (\%). Comparisons of continuous variables among the three groups were carried out using one-way analysis of variance (ANOVA) test continued with post hoc analysis (LSD-t test) or nonparametric test when data did not follow normal distribution 
or homogeneity of variance. Categorical variables were compared using chi-square tests. P-values <.05 (two-sided) were considered statistically significant.

\section{RESULTS}

\section{Demographics, Socioeconomics, and Baseline Clinical Characteristics}

Median time of treatment was 8 (range 5-14) days for eltrombopag and 7 (range 3-14) days for rhTPO. Patients in the control group were followed for a median of 12 (range 7-17) days.

Table 1 shows the demographic, socioeconomic, and clinical features by treatment cohorts. The three groups did not differ with regard to gender, age, and ethnicity. No significant difference was observed for socioeconomic variables including type of medical insurance, monthly household income, education level, and occupation $(\mathrm{P}>.05)$. Clinical characteristics that include BMI, ECOG score, type of lymphoma, disease duration, bone marrow (BM) invasion, chemotherapy regimen, radiation therapy, prechemotherapy platelets, leukocyte counts, hemoglobin, and previous bleeding were also similar among the three groups.

\section{Treatment Efficacy Platelet Response}

Mean platelet counts at baseline (Day 0) were not different among the three groups [eltrombopag: $(23.96 \pm 14.15) \times 10^{9} / \mathrm{L}$, rhTPO: $(23.92 \pm 12.45) \times 10^{9} / \mathrm{L}$, control: $\left.(24.15 \pm 7.47) \times 10^{9} / \mathrm{L} ; \mathrm{P}=.711\right]$. At Day 5, Day 7, and Day 10, significantly higher platelet counts were observed in both the eltrombopag group $\left[(44.24 \pm 17.51) \times 10^{9} / \mathrm{L}\right.$, $\left.(67.30 \pm 29.90) \times 10^{9} / \mathrm{L},(130.73 \pm 70.57) \times 10^{9} / \mathrm{L} ; \mathrm{P}=.003, .000, .000\right]$ and rhTPO group $\left[(48.92 \pm 32.46) \times 10^{9} / \mathrm{L},(82.11 \pm 33.37) \times 10^{9} / \mathrm{L}\right.$, $\left.(147.02 \pm 68.47) \times 10^{9} / \mathrm{L} ; \mathrm{P}=.005, .000, .000\right]$ than the control group $\left[(33.73 \pm 24.62) \times 10^{9} / \mathrm{L},(41.58 \pm 21.27) \times 10^{9} / \mathrm{L},(75.67 \pm\right.$ 40.40) $\left.\times 10^{9} / \mathrm{L}\right]$, but there was no difference between treatment with eltrombopag and $\operatorname{rhTPO}(\mathrm{P}=1.000, .187, .598$; Table 2 and Figure 1). Similar trend was reflected in the lowest platelet count, which was significantly lower in the control group than the eltrombopag group and the rhTPO group $\left[(11.37 \pm 7.66) \times 10^{9} / \mathrm{L}\right.$ vs $\left.(15.94 \pm 9.09) \times 10^{9} / \mathrm{L},(18.28 \pm 15.59) \times 10^{9} / \mathrm{L} ; \mathrm{P}=.041, .005\right]$, but no difference was seen between the two treatment groups $(\mathrm{P}=1.000)$.

Platelet counts $<50 \times 10^{9} / \mathrm{L}$ lasted for $6.25 \pm 2.61$ days in the eltrombopag group and $5.48 \pm 2.62$ days in the rhTPO group $(\mathrm{P}=.599)$, both of which were significantly shorter than the control group $[(8.33 \pm 3.98)$ days; $\mathrm{P}=.036, .000]$. Days required for the recovery of platelet counts to $50 \times 10^{9} / \mathrm{L}$ or higher was comparable between patients treated with eltrombopag and those with rhTPO $(\mathrm{P}=.508)$ but significantly shorter than the control group $[(6.33 \pm$ 2.31) days, $(5.44 \pm 2.57)$ days $v s(8.32 \pm 2.53)$ days; $\mathrm{P}=.001, .000]$. The findings were similar with regard to the days required for the recovery of platelet counts to $\geq 75 \times 10^{9} / \mathrm{L}$ (Table 3 ).

\section{Bleeding Outcomes}

WHO grade 1 bleeding occurred in 3 patients (5.9\%) treated with eltrombopag, 3 patients (6.0\%) with rhTPO, and $8(15.4 \%)$ patients in the control group, whereas grade 2 or 3 bleeding occurred in $3(5.9 \%)$ patients treated with eltrombopag, 2 (4.0\%) patients with rhTPO, and $6(11.5 \%)$ patients in the control group (Figure 2). No cases of grade 4 bleeding occurred during study. Rates of overall bleeding (any grades 1-4) were significantly higher in the control group than eltrombopag and rhTPO groups (26.9\% vs $11.8 \%, 10.0 \% ; \mathrm{P}=.031, .017$ ), yet there was no statistical difference between treatment with eltrombopag and rhTPO $(\mathrm{P}=.776$; Table 4).

\section{Platelet Transfusion}

A total of 28 (54.9\%), 25 (50.0\%), and 39 (75.0\%) patients received one unit of platelet transfusion in the eltrombopag group, the rhTPO group, and the control group, respectively. Compared to the two treatment groups, a significantly higher proportion of cases required platelet transfusion in the control group $(\mathrm{P}<.05$; Table 4). Platelet transfusion rates did not differ between eltrombopag and rhTPO treatment $(\mathrm{P}=.622)$.

\section{Safety and Tolerability}

Seven patients (13.7\%) experienced AEs that may be related to eltrombopag treatment, including 3 cases of elevated transaminase (5.9\%) and 1 case each (2.0\%) of hyperbilirubinemia, fever, fatigue, and dizziness. Six patients (12.0\%) experienced AEs that may be related to rhTPO treatment, including 2 cases of fever (4.0\%) and 1 case each $(2.0 \%)$ of fatigue, dizziness, diarrhea, and muscle aches. All these AEs were mild and transient in nature. The elevation of transaminase and blood bilirubin in the eltrombopag group were resolved after a short-term liver protection treatment, while the other AEs were relieved spontaneously without special treatment. No serious AEs were reported. Overall, eltrombopag and rhTPO were well tolerated in our study (Table 5).

\section{DISCUSSION}

In China, rhTPO was recommended for the treatment of severe CIT and as a prophylactic option in cancer patients with high risk of bleeding after chemotherapy $(9,10)$. The efficacy and safety of rhTPO to treat CIT in patients with solid tumor have been well demonstrated in previous studies $(16,17)$. Eltrombopag was reported as an effective agent to maintain platelet level, reduce bleeding episodes and transfusion requirements, and alleviate chemotherapy dose reductions and delays without compromising patient safety in a number of small-sample, early phase trials $(6,12-$ 14, 18-21). Due to limited data available, eltrombopag has not been approved for the treatment of CIT; however, in view of the difficulties of CIT treatment and seriousness of bleeding consequences, it is considered as an alternative therapeutic option for use in patients with poor response to rhTPO (10). This is the first study to systematically evaluate and compare the effectiveness and safety of eltrombopag versus rhTPO for treatment of CIT in patients with lymphoma. Based on a large cohort of patients, our findings provide summarized experience in a real-world clinical practice.

Cytotoxic drugs used in chemotherapy can lead to increased platelet destruction, reduced platelet production, and abnormal platelet distribution that result in thrombocytopenia (22). TPO- 
TABLE 1 | Demographic, socioeconomic, and baseline characteristics.

\begin{tabular}{|c|c|c|c|c|}
\hline & Eltrombopag $(\mathrm{N}=51)$ & rhTPO (N=50) & Control $(\mathrm{N}=52)$ & P value $^{\mathbf{a}}$ \\
\hline Male, n (\%) & $29(56.9)$ & $30(60.0)$ & $31(59.6)$ & .941 \\
\hline \multicolumn{5}{|l|}{ Age (years), } \\
\hline Mean \pm SD & $49.1 \pm 18.7$ & $50.8 \pm 15.3$ & $48.3 \pm 18.2$ & .865 \\
\hline Range & $15-86$ & $25-87$ & $17-80$ & \\
\hline Ethnic group, n (\%) & & & & $1.000^{b}$ \\
\hline Han & $51(100.0)$ & $50(100.0)$ & $51(98.1)$ & \\
\hline Others & $0(0.0)$ & $0(0.0)$ & $1(1.9)$ & \\
\hline Medical insurance, n (\%) & & & & $.863^{\mathrm{b}}$ \\
\hline Urban employees' basic medical insurance & $10(19.6)$ & $13(26.0)$ & $15(28.8)$ & \\
\hline Urban residents' basic medical insurance & $17(33.3)$ & $19(38.0)$ & $12(23.1)$ & \\
\hline New rural cooperative medical system & $7(13.7)$ & $5(10.0)$ & $9(17.3)$ & \\
\hline Mixed medical insurance ${ }^{c}$ & $4(7.8)$ & $2(4.0)$ & $2(3.8)$ & \\
\hline Full coverage $^{d}$ & $2(3.9)$ & $4(8.0)$ & $3(5.8)$ & \\
\hline Others $^{\mathrm{e}}$ & $3(5.9)$ & $3(6.0)$ & $4(7.7)$ & \\
\hline Uninsured & $8(15.7)$ & $4(8.0)$ & $7(13.5)$ & \\
\hline Household income (¥/month) ${ }^{f}, \mathrm{n}(\%)$ & & & & $.949^{b}$ \\
\hline$<5000$ & $18(35.3)$ & $14(28.0)$ & $19(36.5)$ & \\
\hline $5000-10000$ & $10(19.6)$ & $15(30.0)$ & $15(28.8)$ & \\
\hline $10001-15000$ & $6(11.8)$ & $8(16.0)$ & $5(9.6)$ & \\
\hline $15001-20000$ & $9(17.6)$ & $7(14.0)$ & $8(15.4)$ & \\
\hline$>20000$ & $5(9.8)$ & $3(6.0)$ & $3(5.8)$ & \\
\hline Unknowng & $3(5.9)$ & $3(6.0)$ & $2(3.8)$ & \\
\hline Education level, n (\%) & & & & .606 \\
\hline Primary school or lower & $17(33.3)$ & $15(30.0)$ & $22(42.3)$ & \\
\hline Middle school & $14(27.5)$ & $13(26.0)$ & $10(19.2)$ & \\
\hline High school & $12(23.5)$ & $15(30.0)$ & $9(17.3)$ & \\
\hline College or above & $8(15.7)$ & $7(14.0)$ & $11(21.2)$ & \\
\hline Occupation, n (\%) & & & & .718 \\
\hline Manual workers & $6(11.8)$ & $10(20.0)$ & $8(15.4)$ & \\
\hline Agricultural workers & $9(17.6)$ & $6(12.0)$ & $10(19.2)$ & \\
\hline Self-employed & $14(27.5)$ & $8(16.0)$ & $9(17.3)$ & \\
\hline Managers and professionals & $10(19.6)$ & $11(22.0)$ & $14(26.9)$ & \\
\hline Unemployed & $12(23.5)$ & $15(30.0)$ & $11(21.2)$ & \\
\hline BMl $\left(\mathrm{kg} / \mathrm{m}^{2}\right)$, mean (range) & $21.1(17.5-26.6)$ & $21.0(17.1-26.1)$ & $20.6(15.4-27.9)$ & .521 \\
\hline ECOG score, mean (range) & $2.9(2-4)$ & $2.7(2-4)$ & $2.9(2-4)$ & .063 \\
\hline Time since diagnosis of lymphoma (months), mean (range) & $16.0(1-120)$ & $18.0(1-60)$ & $14.0(1-72)$ & .856 \\
\hline Type of lymphoma, $\mathrm{n}(\%)$ & & & & $1.000^{\mathrm{b}}$ \\
\hline DLBCL & $28(54.9)$ & $26(52.0)$ & $28(53.9)$ & \\
\hline T or B lymphoblastic lymphoma & $7(13.7)$ & $7(14.0)$ & $8(15.4)$ & \\
\hline NK/T-cell lymphoma & $5(9.8)$ & $5(10.0)$ & $4(7.7)$ & \\
\hline PTCL & $3(5.9)$ & $4(8.0)$ & $4(7.7)$ & \\
\hline Burkitt lymphoma & $6(11.8)$ & $6(12.0)$ & $5(9.6)$ & \\
\hline Other types ${ }^{h}$ & $2(3.9)$ & $2(4.0)$ & $3(5.8)$ & \\
\hline Bone marrow invasion of lymphoma, n (\%) & $5(9.8)$ & $5(10.0)$ & $6(11.5)$ & .952 \\
\hline Chemotherapy, n (\%) & & & & $1.000^{\mathrm{b}}$ \\
\hline $\mathrm{CHOP}$ or $\mathrm{CDOP} \pm \mathrm{R}$ & $23(45.1)$ & $22(44.0)$ & $25(48.1)$ & \\
\hline $\mathrm{R}-\mathrm{EPOCH}$ & $3(5.9)$ & $3(4.0)$ & $3(5.8)$ & \\
\hline R-CODOX-M or R-IVAC & $6(11.8)$ & $6(14.0)$ & $5(7.7)$ & \\
\hline CAM & $3(5.9)$ & $4(8.0)$ & $4(7.7)$ & \\
\hline VDLP & $4(7.8)$ & $3(6.0)$ & $4(7.7)$ & \\
\hline P-Gemox & $5(9.8)$ & $5(10.0)$ & $4(9.6)$ & \\
\hline DICE & $4(7.8)$ & $4(8.0)$ & $3(5.8)$ & \\
\hline Other chemotherapy ${ }^{i}$ & $3(5.9)$ & $3(6.0)$ & $4(7.7)$ & \\
\hline Prior chemotherapy regimens, mean (range) & $6.1(1-20)$ & $6.6(1-19)$ & $6.9(1-16)$ & .518 \\
\hline Radiation therapy, n (\%) & $3(5.9)$ & $3(6.0)$ & $2(3.9)$ & .860 \\
\hline \multicolumn{5}{|l|}{ Platelet count before chemotherapy $\left(\times 10^{9} / \mathrm{L}\right)$} \\
\hline Mean \pm SD & $135.7 \pm 129.1$ & $130.3 \pm 78.7$ & $134.7 \pm 87.1$ & .512 \\
\hline Range & $5-625$ & $7-309$ & $8-370$ & \\
\hline \multicolumn{5}{|l|}{ Leukocyte count ( $\left.\times 10^{9} / \mathrm{L}\right)$} \\
\hline Mean \pm SD & $2.5 \pm 2.7$ & $2.5 \pm 2.5$ & $2.6 \pm 3.5$ & .469 \\
\hline Range & $0.14-11.19$ & $0.05-12.11$ & $0.08-16.54$ & \\
\hline
\end{tabular}


TABLE 1 | Continued

\begin{tabular}{|c|c|c|c|c|}
\hline & Eltrombopag ( $\mathrm{N}=51)$ & rhTPO $(\mathrm{N}=50)$ & Control $(\mathrm{N}=52)$ & P value ${ }^{a}$ \\
\hline \multicolumn{5}{|l|}{ Hemoglobin (g/L) } \\
\hline Mean \pm SD & $75.0 \pm 18.0$ & $78.7 \pm 17.7$ & $78.7 \pm 22.7$ & .457 \\
\hline Previous bleeding, $\mathrm{n}(\%)$ & $2(3.9)$ & $3(6.0)$ & $2(3.8)$ & .798 \\
\hline
\end{tabular}

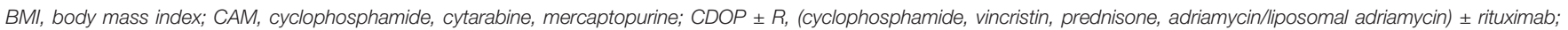

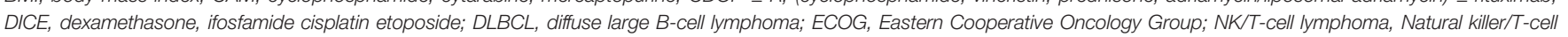
lymphoma; P-Gemox, peaspartase, gemcitabine, oxaliplatin; PTCL, peripheral T-cell lymphoma; R-CODOX-M, tuximab, cyclophosphamide, vincristine, adriamycin, methotrexate;

R-IVAC, tuximab, ifosfamide, etoposide, arabinoside glycoside; VDLP, prednisone, daunorubicin or liposomal adriamycin, vincristine, pegaspase.

${ }^{a} P$ value compares baseline characteristics among three groups.

${ }^{b}$ Using Fisher's exact test in $R^{*} C$ table Chi-Square Test.

${ }^{c}$ Two or more insurances, normally indicating the basic insurance plus other supplementary insurances in China.

${ }^{d}$ Medical costs can be almost fully covered.

${ }^{e}$ Other single insurance.

f $¥ 5000$ equals to US\$775, ¥5000-10000 equals to US\$775-1550, ¥10001-15000 equals to US\$1550-2325, ¥15001-20000 equals to US\$2325-3100, ¥20000 equals to US\$3100. ${ }^{g}$ Patients either refused to answer the question or did not know the answer.

hOther types of lymphoma include marginal area lymphoma, follicular lymphoma and mantle cell lymphoma.

'Other chemotherapy include GDP (gemcitabine, cisplatin, dexamethasone), BR (bendamustine, rituximab), and R+MTX (rituximab, methotrexate).

${ }^{j}$ WHO bleeding grades 1 or 2 .

RAs were designed as mimics to endogenous TPO that regulates the whole process of megakaryopoiesis and promotes platelet production $(3,23,24)$. rhTPO, as the first generation TPO-RA, was shown to be effective in alleviating and shortening the duration of CIT at a daily dose of $15,000 \mathrm{U}$ in our patients with lymphoma. Eltrombopag is the second generation, nonpeptide TPO-RA that binds to the transmembrane domain of the thrombopoietin receptor and increases platelet counts by stimulating megakaryocytes proliferation from BM progenitor cells (25). Unlike rhTPO, eltrombopag does not compete with or elicit an antibody response to endogenous TPO (26). At a starting dose of $50 \mathrm{mg}$ and adjustable daily dose during treatment, our results indicated that eltrombopag was comparable to rhTPO in terms of the efficacy to increase platelet counts and nadir platelet counts, and reduce the time required for platelet recovery.

In a study of rhTPO for the treatment of CIT in patients with solid tumor, time required for platelet levels recovered to $\geq 75 \times 10^{9} /$ $\mathrm{L}$ and $\geq 100 \times 10^{9} / \mathrm{L}$ were $4.79 \pm 3.67$ and $6.93 \pm 3.61$ days, respectively, among 72 patients who had a post-chemotherapy platelet count of $55.9 \pm 16.0\left(\times 10^{9} / \mathrm{L}\right)(27)$. Another randomized, cross-over, self-controlled trial of rhTPO reported a mean of $2.5 \pm$ $3.9,10.3 \pm 8.7$, and $15.9 \pm 10.5$ days with respect to the duration of thrombocytopenia (platelet counts $<50 \times 10^{9} / \mathrm{L}$ ), days required for the recovery of platelet counts to $\geq 75 \times 10^{9} / \mathrm{L}$ and $\geq 100 \times 10^{9} / \mathrm{L}$, respectively (17). Our findings on the days

TABLE 2 | Platelet counts among the three groups.

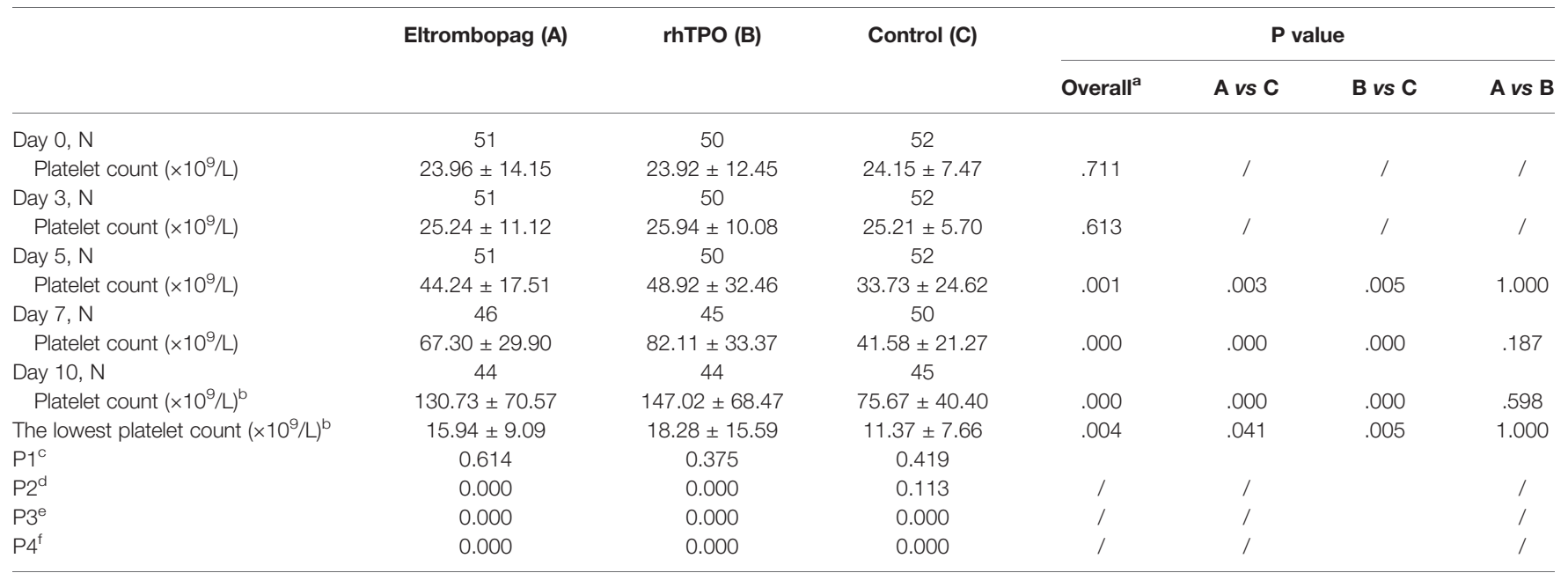

Data are mean $\pm S D$.

${ }^{a} P$ value compares platelet counts among the three groups.

${ }^{b}$ Defined as the lowest platelet count observed after treatment initiation (or after enrolment for patients of the control group).

${ }^{c} P$ value compares platelet counts between Day 0 and Day 3.

${ }^{d} P$ value compares platelet counts between Day 0 and Day 5.

${ }^{e} P$ value compares platelet counts between Day 0 and Day 7.

${ }^{f} P$ value compares platelet counts between Day 0 and Day 10. 


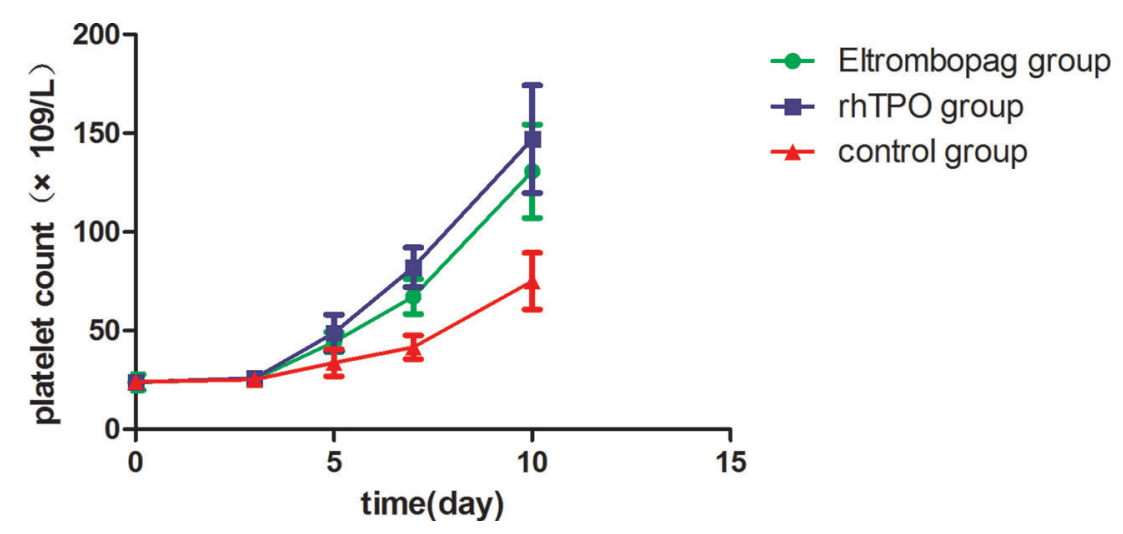

FIGURE 1 | Mean platelet counts during treatment period among the three groups. Error bars indicate $95 \% \mathrm{Cl}$ of the mean. $\mathrm{Cl}$, confidence interval.

TABLE 3 | Platelet response among the three groups.

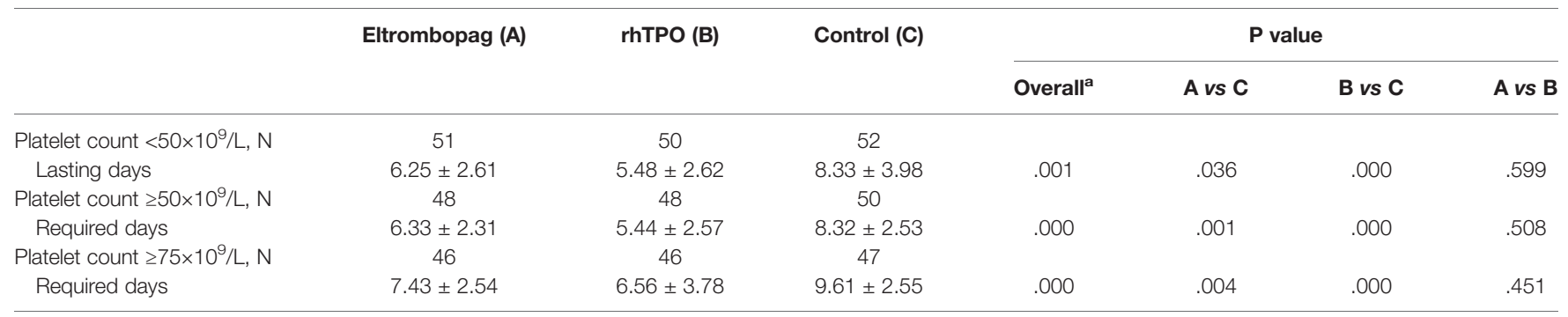

Data are mean $\pm S D$.

${ }^{a} P$ value compares data among the three groups.

required for the recovery of platelet counts to $\geq 75 \times 10^{9} / \mathrm{L}$ [eltrombopag $(7.43 \pm 2.54)$ days, $\operatorname{rhTPO}(6.56 \pm 3.78)$ days; Table 3] were comparable to the aforementioned studies, yet the platelet counts $<50 \times 10^{9} / \mathrm{L}$ lasting days were observed to be longer in either of our treatment groups [eltrombopag $(6.25 \pm 2.61)$ days, rhTPO (5.48 \pm 2.62$)$ days; Table 3]. This might be explained by the presence of more severe thrombocytopenia at baseline in our patient population, as subgroup analysis of severe CIT in the study of Bai et al. (16) reported longer duration of platelet counts $<50 \times 10^{9} / \mathrm{L}$ (median 11 days) and that median time required for the recovery of platelet counts to $\geq 75 \times 10^{9} / \mathrm{L}$ and $\geq 100 \times 10^{9} / \mathrm{L}$ were 21 and 24 days, respectively. Due to the real-world nature of this

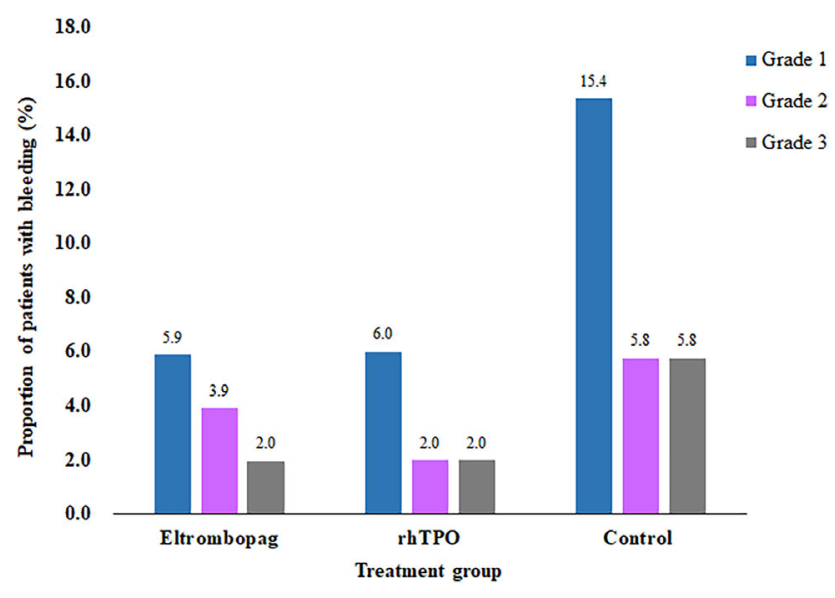

FIGURE 2 | Proportion of bleeding by WHO grades among the three groups. 
TABLE 4 | Bleeding by WHO grades and platelet transfusion after treatment.

\begin{tabular}{|c|c|c|c|c|c|c|c|}
\hline & \multirow{2}{*}{$\begin{array}{l}\text { Eltrombopag (A) } \\
(\mathrm{N}=51)\end{array}$} & \multirow{2}{*}{$\begin{array}{l}\text { rhTPO (B) } \\
(\mathrm{N}=50)\end{array}$} & \multirow{2}{*}{$\begin{array}{l}\text { Control (C) } \\
(\mathrm{N}=52)\end{array}$} & \multicolumn{4}{|c|}{$P$ value } \\
\hline & & & & Overall $^{a}$ & A vs $C$ & B vs C & $A$ vs $B$ \\
\hline Any bleeding (Grades 1-4), n (\%) & $6(11.8)$ & $5(10.0)$ & $14(26.9)$ & .019 & .031 & .017 & .776 \\
\hline Platelet transfusion, n (\%) & $28(54.9)$ & $25(50.0)$ & $39(75.0)$ & .023 & .032 & .009 & .622 \\
\hline
\end{tabular}

${ }^{a} P$ value compares data among the three groups.

study, some patients gave up the treatment due to financial burden such that we did not have adequate data to calculate days required for the recovery of platelet counts to $\geq 100 \times 10^{9} / \mathrm{L}$. Well controlled, prospective studies will be conducted in the future to observe platelet response in a longer period with more comprehensive follow-up schedules.

CIT increases the risk of bleeding that may lead to dose adjustment and delayed treatment schedules with chemotherapy $(22,28)$. It was studied in cancer patients after chemotherapy that when platelet counts fell below $50 \times 10^{9} / \mathrm{L}$, probability of any bleeding ranges between 0-9.6\%; however, the risk doubles when platelet counts $<20 \times 10^{9} / \mathrm{L}(10.1-17.7 \%)$ and continues to double when $<10 \times 10^{9} / \mathrm{L}(18.4-40.1 \%)(7)$. Our study revealed that eltrombopag or rhTPO treatment was associated with significantly reduced rates of any bleeding and clinically significant bleeding (grade 2-4) when compared to the control group. In a study of patients with immune thrombocytopenia (ITP), regardless of platelet response, a reduced proportion of bleeding episodes were observed during eltrombopag treatment, which was considered to be correlated with the platelet adhesion effect enhanced by eltrombopag (29). Apart from a low baseline platelet count, a previous bleeding episode, BM metastasis, poor ECOG score $(\geq 2)$, previous radiotherapy, and special chemotherapy regimen (cisplatin, carboplatin, gemcitabine, carmustine, or lomustine) were all related to elevated risk of bleeding in patients with CIT (30). Our findings showed no difference of bleeding rates between eltrombopag and rhTPO treatment groups; meanwhile, baseline predictors of bleeding were similar between the two groups, indicating that eltrombopag can be as effective as rhTPO with respect to reducing the risk and severity of bleeding events associated with CIT in patients with lymphoma.

Platelet transfusion is the elective procedure for prevention and treatment of bleeding in patients with hematological disorders, chemotherapy or hematopoietic stem cell transplantation. In case of an active bleeding, it is the first line

TABLE 5 | Treatment-related adverse events.

\begin{tabular}{lcc}
\hline & Eltrombopag (N=51) & rhTPO (N=50) \\
\hline Any AE, n (\%) & $7(13.7)$ & $6(12.0)$ \\
Elevated transaminase & $3(5.9)$ & $0(0.0)$ \\
Hyperbilirubinemia & $1(2.0)$ & $0(0.0)$ \\
Fever & $1(2.0)$ & $2(4.0)$ \\
Fatigue & $1(2.0)$ & $1(2.0)$ \\
Dizziness & $1(2.0)$ & $1(2.0)$ \\
Diarrhea & $0(0.0)$ & $1(2.0)$ \\
Muscle aches & $0(0.0)$ & $1(2.0)$
\end{tabular}

of therapy if bleeding is considered associated with CIT (31). Prophylactic platelet transfusions are indicated when bleeding occurs or when platelet counts are $<10 \times 10^{9} / \mathrm{L}$ ( or $<20 \times 10^{9} / \mathrm{L}$ if the patient is febrile) (3). However, repeated transfusions may lead to problems such as refractoriness, alloimmunization, febrile reactions, and transmission of infectious agents $(7,32,33)$. Our study revealed significantly lower frequencies and volume of platelet transfusion in patients treated with eltrombopag or rhTPO when compared to the controls, but they were not different between eltrombopag and rhTPO treatment. When rhTPO was administered to patients with gynecologic cancer who developed severe thrombocytopenia after carboplatin chemotherapy, the need for platelet transfusion was reduced from the pre-treatment rate of $75 \%$ to $25 \%(P=.013)(34)$. Since it requires for about 5 days for platelet counts to rise after administering TPO-RAs, platelet transfusion, if indicated, should be used together with TPO-RAs (3). By stimulating platelet production that eventually leads to elevated platelet counts $(24,25)$, TPO-RAs such as eltrombopag and rhTPO can reduce the need for platelet transfusion due to bleeding or low platelet counts, thus lowering the overall risk of transfusion reactions and non-response.

In general, eltrombopag and rhTPO were well tolerated as all of the treatment-related AEs observed in our study were mild and in accordance with the safety profiles of previous reports (16, $19,33,35)$. Hepatobiliary toxicity is a major concern associated with eltrombopag use in patients of east Asian descent $(20,36)$. Elevated transaminase and hyperbilirubinemia occurred in $5.9 \%$ and $2.0 \%$ of our patients treated with eltrombopag, respectively. All these hepatobiliary abnormalities were mild and resolved after a short-term liver protection treatment. In a phase II study assessing the efficacy and safety of eltrombopag in patients receiving carboplatin/paclitaxel for treatment of advanced solid tumors, elevated aminotransferase $\geq 3$ times the ULN and total bilirubin $\geq 1.5$ times the ULN were $11 \%$ and $18 \%$ in the $50 \mathrm{mg}$ group, $17 \%$ and $14 \%$ in the $75 \mathrm{mg}$ group, and $13 \%$ and $23 \%$ in the $100 \mathrm{mg}$ group (12). Hepatobiliary AEs were reported in 19\% patients in a phase $1 / 2$ trial assessing the safety and tolerability of eltrombopag for treatment of thrombocytopenia in patients with advanced myelodysplastic syndromes or acute myeloid leukemia, including $8 \%$ with grade 3 or higher events (14). Our rates and severity of elevated hepatobiliary values were lower than these findings, suggesting that eltrombopag can be safely prescribed to treat CIT in patients with lymphoma.

Due to the good tolerability and efficacy to reduce the incidence and duration of thrombocytopenia, rhTPO was proposed as a second-line treatment option for CIT by the 
Chinese Society of Clinical Oncology $(9,10)$. Our preliminary findings on a relatively large sample of patients with lymphoma suggested that eltrombopag had comparable efficacy and safety with rhTPO for treatment of CIT. Injection site reactions such as pain and ecchymosis are commonly expected in patients treated with rhTPO as the drug was approved for subcutaneous use. Eltrombopag, developed as an oral preparation, allows to avoid injection-related reactions and is considered more convenient for patients to administer. Well-designed medical cost research studies conducted in a wider geographic area of China are needed to further support if eltrombopag, when compared to rhTPO, is also a cost-effective treatment option to lymphoma patients with CIT.

There are several limitations in our study: first, because of retrospective and observational design, we are unable to evaluate how eltrombopag and rhTPO administration help to maintain scheduled dosing and treatment cycles of chemotherapy. Secondly, as this is a single-center study, generalizability of study results to other population should be made with caution. Thirdly, due to the inclusion of multiple chemotherapy regimens, it may not be possible to establish the impact of eltrombopag and rhTPO treatment on CIT after a specific regimen. Also, prognostic outcomes, such as progression-free and overall survival, cannot be explored owning to the relatively short duration of follow-up in the current study. Further prospective, interventional studies are needed to investigate the long-term effectiveness and safety of eltrombopag and rhTPO for treatment of CIT in patients with lymphoma that receive myelosuppressive chemotherapy, and to identify the optimal dose and dosing frequencies.

In conclusion, both eltrombopag and rhTPO were effective in the treatment of thrombocytopenia after chemotherapy for lymphoma with respect to the elevated platelet counts, prolonged periods of platelet response, reduced bleeding episodes and platelet transfusion requirements. Eltrombopag was well tolerated in real-world setting without raising additional concerns for hepatobiliary toxicity. Evidence from

\section{REFERENCES}

1. Shankland KR, Armitage JO, Hancock BW. Non-Hodgkin Lymphoma. Lancet (2012) 380:848-57. doi: 10.1016/S0140-6736(12)60605-9

2. Bjorn N, Sigurgeirsson B, Svedberg A, Pradhananga S, Brandén E, Koyi H, et al. Genes and Variants in Hematopoiesis-Related Pathways are Associated With Gemcitabine/Carboplatin-Induced Thrombocytopenia. Pharmacogenomics J (2020) 20:179-91. doi: 10.1038/s41397-019-0099-8

3. Kuter DJ. Managing Thrombocytopenia Associated With Cancer Chemotherapy. Oncol (Williston Park) (2015) 29:282-94.

4. Wu Y, Aravind S, Ranganathan G, Martin A, Nalysnyk L. Anemia and Thrombocytopenia in Patients Undergoing Chemotherapy for Solid Tumors: A Descriptive Study of a Large Outpatient Oncology Practice Database, 20002007. Clin Ther (2009) 31:2416-32. doi: 10.1016/j.clinthera.2009.11.020

5. Weycker D, Hatfield M, Grossman A, Hanau A, Lonshteyn A, Sharma A, et al. Risk and Consequences of Chemotherapy-Induced Thrombocytopenia in US Clinical Practice. BMC Cancer (2019) 19:151. doi: 10.1186/s12885-019-5354-5

6. Winer ES, Safran H, Karaszewska B, Bauer S, Khan D, Doerfel S, et al. Eltrombopag for Thrombocytopenia in Patients With Advanced Solid Tumors Receiving Gemcitabine-Based Chemotherapy: A Randomized, Placebo-Controlled Phase 2 Study. Int J Hematol (2017) 106:765-76. doi: $10.1007 / \mathrm{s} 12185-017-2319-9$ this large cohort study supports the use of eltrombopag as an alternative treatment option for CIT in lymphoma patients.

\section{DATA AVAILABILITY STATEMENT}

The original contributions presented in the study are included in the article/supplementary material. Further inquiries can be directed to the corresponding author.

\section{ETHICS STATEMENT}

The studies involving human participants were reviewed and approved by Ethics Committee of the First Affiliated Hospital of Guangdong Pharmaceutical University. Written informed consent for participation was not required for this study in accordance with the national legislation and the institutional requirements.

\section{AUTHOR CONTRIBUTIONS}

QZ, SY, and XP conceptualized and designed the study. QZ and SY collected and analyzed data, and drafted the paper. LZ, WZ, and XP carried out the data analysis, and revised the paper. All authors contributed to the article and approved the submitted version.

\section{FUNDING}

This study was supported by the Science and Technology Planning Project of Guangzhou (202002030253), the Natural Science Foundation of Guangdong Province (2017A030313664), the Science and Technology Project of Yuexiu District of Guangzhou (2017-W S-008).

7. Zhang X, Chuai Y, Nie W, Wang A, Dai G. Thrombopoietin Receptor Agonists for Prevention and Treatment of Chemotherapy-Induced Thrombocytopenia in Patients With Solid Tumors. Cochrane Database Syst Rev (2017) 11:CD12035. doi: 10.1002/14651858.CD012035.pub2

8. Kuter DJ. The Biology of Thrombopoietin and Thrombopoietin Receptor Agonists. Int J Hematol (2013) 98:10-23. doi: 10.1007/s12185-013-1382-0

9. Chinese Society of Clinical Oncology (CSCO) Anti-Lymphoma Alliance, Chinese Society of Clinical Oncology (CSCO) Anti-Leukemia Alliance. [Chinese Expert Consensus on Prevention and Treatment of Chemotherapy-Induced Thrombocytopenia in Malignant Lymphoma]. J Leukemia Lymphoma (2020) 29:65-72. doi: 10.3760/cma.j.issn.10099921.2020.02.001

10. The Society of Chemotherapy, China Anti-Cancer Association; Committee of Neoplastic Supportive-Care (CONS), China Anti-Cancer Association. Consensus on the Clinical Diagnosis, Treatment, and Prevention of Chemotherapy-Induced Thrombocytopenia in China (2019 Version). Chin J Front Med Sci (Electronic Version) (2020) 12:51-8. doi: 10.12037/ YXQY.2020.01-07

11. Nevil G, Roth M, Gill J, Zhang W, Teicher B, Erickson SW, et al. Initial In Vivo Testing of TPO-Receptor Agonist Eltrombopag in Osteosarcoma PatientDerived Xenograft Models by the Pediatric Preclinical Testing Consortium. Pediatr Hematol Oncol (2021) 38:8-13. doi: 10.1080/08880018.2020.1802539 
12. Kellum A, Jagiello-Gruszfeld A, Bondarenko IN, Patwardhan R, Messam C, Mostafa Kamel Y. A Randomized, Double-Blind, Placebo-Controlled, Dose Ranging Study to Assess the Efficacy and Safety of Eltrombopag in Patients Receiving Carboplatin/Paclitaxel for Advanced Solid Tumors. Curr Med Res Opin (2010) 26:2339-46. doi: 10.1185/03007995.2010.510051

13. Winer ES, Safran H, Karaszewska B, Richards DA, Hartner L, Forget F, et al. Eltrombopag With Gemcitabine-Based Chemotherapy in Patients With Advanced Solid Tumors: A Randomized Phase I Study. Cancer Med (2015) 4:16-26. doi: $10.1002 / \mathrm{cam} 4.326$

14. Platzbecker U, Wong RS, Verma A, Abboud C, Araujo S, Chiou TJ, et al. Safety and Tolerability of Eltrombopag Versus Placebo for Treatment of Thrombocytopenia in Patients With Advanced Myelodysplastic Syndromes or Acute Myeloid Leukaemia: A Multicentre, Randomised, PlaceboControlled, Double-Blind, Phase 1/2 Trial. Lancet Haematol (2015) 2:e417426. doi: 10.1016/S2352-3026(15)00149-0

15. National Cancer Institute. Common Terminology Criteria for Adverse Events (CTCAE). Version 5.0. Available at: https://ctep.cancer.gov/protocoldevelopment/ electronic_applications/ctc.htm\#ctc_50 (Accessed 14 April (2021)). valid from 2017-11-27.

16. Bai CM, Zou XY, Zhao YQ, Han SM, Shan YD. The Clinical Study of Recombinant Human Thrombopoietin in the Treatment of ChemotherapyInduced Severe Thrombocytopenia. Natl Med J China (2004) 84:397. doi: 10.3760/j:issn:0376-2491.2004.05.013

17. Bai CM, Xu GX, Zhao YQ, Han SM, Shan YD. A Multi-Center Clinical Trial of Recombinant Human Thrombopoietin in the Treatment of Chemotherapy -Induced Thrombocytopenia in Patients With Solid Tumor. Zhong guo Yi Xue Ke Xue Yuan Xue Bao (2004) 26:437-41. doi: 10.3321/j.issn:1000503X.2004.04.020

18. Chawla SP, Staddon A, Hendifar A, Messam CA, Patwardhan R, Kamel YM. Results of a Phase I Dose Escalation Study of Eltrombopag in Patients With Advanced Soft Tissue Sarcoma Receiving Doxorubicin and Ifosfamide. BMC Cancer (2013) 13:121. doi: 10.1186/1471-2407-13-121

19. Mukherjee S, Elson P, Bogati S, Woltman A, Desamito J, Gerds AT, et al. A Single Arm, Phase II Study of Eltrombopag to Enhance Platelet Count Recovery in Older Patients With AML Undergoing Remission Induction Therapy. Blood (2016) 128:447. doi: 10.1182/blood.V128.22.447.447

20. Mittelman M, Platzbecker U, Afanasyev B, Grosicki S, Wong RSM, Anagnostopoulos A, et al. Eltrombopag for Advanced Myelodysplastic Syndromes or Acute Myeloid Leukaemia and Severe Thrombocytopenia (ASPIRE): A Randomised, Placebo-Controlled, Phase 2 Trial. Lancet Haematol (2018) 5:e34-43. doi: 10.1016/S2352-3026(17)30228-4

21. Frey N, Jang JH, Szer J, Illés Á, Kim HJ, Ram R, et al. Eltrombopag Treatment During Induction Chemotherapy for Acute Myeloid Leukaemia: A Randomised, Double-Blind, Phase 2 Study. Lancet Haematol (2019) 6:e12231. doi: 10.1016/S2352-3026(18)30231-X

22. Razzaghdoust A, Mofid B, Zangeneh M. Predicting Chemotherapy-Induced Thrombocytopenia in Cancer Patients With Solid Tumors or Lymphoma. J Oncol Pharm Pract (2020) 26:587-94. doi: 10.1177/1078155219861423

23. Osada M, Komeno T, Todokoro K, Takizawa M, Kojima H, Suzukawa K, et al. Immature Megakaryocytes Undergo Apoptosis in the Absence of Thrombopoietin. Exp Hematol (1999) 27:131-8. doi: 10.1016/s0301-472x (98)00007-1

24. Saleh MN, Bussel JB, Cheng G, Meyer O, Bailey CK, Arning M, et al. Safety and Efficacy of Eltrombopag for Treatment of Chronic Immune Thrombocytopenia: Results of the Long-Term, Open-Label EXTEND Study. Blood (2013) 121:537-45. doi: 10.1182/blood-2012-04-425512

25. Kuter DJ. New Thrombopoietic Growth Factors. Blood (2007) 109(11):460716. doi: 10.1182/blood-2006-10-019315
26. Basciano PA, Bussel JB. Thrombopoietin-Receptor Agonists. Curr Opin Hematol (2012) 19:392-8. doi: 10.1097/MOH.0b013e328356e909

27. Dai XF, Yu J, Liu L, Wu G. Value of Recombinant Human Thrombopoietin in the Treatment of Chemotherapy-Induced Thrombocytopenia With Solid Tumor. Zhonghua Zhong Liu Za Zhi (2008) 30:623-5. doi: 10.3321/ j.issn:0253-3766.2008.08.016

28. Al-Samkari H, Parnes AD, Goodarzi K, Weitzman JI, Connors JM, Kuter DJ. A Multicenter Study of Romiplostim for Chemotherapy-Induced Thrombocytopenia in Solid Tumors and Hematologic Malignancies. Haematologica (2021) 106:1148-57. doi: 10.3324/haematol.2020.251900

29. Bussel J, Kulasekararaj A, Cooper N, Verma A, Steidl U, Semple JW, et al. Mechanisms and Therapeutic Prospects of Thrombopoietin Receptor Agonists. Semin Hematol (2019) 56:262-78. doi: 10.1053/j.seminhematol.2019.09.001

30. Elting LS, Rubenstein EB, Martin CG, Kurtin D, Rodriguez S, Laiho E, et al. Incidence, Cost, and Outcomes of Bleeding and Chemotherapy Dose Modification Among Solid Tumor Patients With Chemotherapy-Induced Thrombocytopenia. J Clin Oncol (2001) 19:1137-46. doi: 10.1200/ JCO.2001.19.4.1137

31. Castaman G, Pieri L. Management of Thrombocytopenia in Cancer. Thromb Res (2018) 164(Suppl 1):S89-93. doi: 10.1097/MOH.0000000000000273

32. Kuter DJ, Begley CG. Recombinant Human Thrombopoietin: Basic Biology and Evaluation of Clinical Studies. Blood (2002) 100:3457-69. doi: 10.1182/ blood.V100.10.3457

33. Xu Y, Song X, Du F, Zhao Q, Liu L, Ma Z, et al. A Randomized Controlled Study of Rhtpo and Rhil-11 for the Prophylactic Treatment of ChemotherapyInduced Thrombocytopenia in Non-Small Cell Lung Cancer. J Cancer (2018) 9:4718-25. doi: 10.7150/jca.26690

34. Vadhan-Raj S, Verschraegen CF, Bueso-Ramos C, Broxmeyer HE, Kudelkà AP, Freedman RS, et al. Recombinant Human Thrombopoietin Attenuates Carboplatin-Induced Severe Thrombocytopenia and the Need for Platelet Transfusions in Patients With Gynecologic Cancer. Ann Intern Med (2000) 132:364-8. doi: 10.7326/0003-4819-132-5-200003070-00005

35. Wang Z, Fang X, Huang H, Hong H, Li X, Guo C, et al. Recombinant Human Thrombopoietin (Rh-TPO) for the Prevention of Severe Thrombocytopenia Induced by High-Dose Cytarabine: A Prospective, Randomized, Self-Controlled Study. Leuk Lymphoma (2018) 59:2821-8. doi: 10.1080/10428194.2018.1459605

36. Yamazaki $\mathrm{H}$, Ohta $\mathrm{K}$, Iida $\mathrm{H}$, Imada $\mathrm{K}$, Obara $\mathrm{N}$, Tokumine $\mathrm{Y}$, et al. Hematologic Recovery Induced by Eltrombopag in Japanese Patients With Aplastic Anemia Refractory or Intolerant to Immunosuppressive Therapy. Int J Hematol (2019) 110:187-96. doi: 10.1007/s12185-019-02683-1

Conflict of Interest: The authors declare that the research was conducted in the absence of any commercial or financial relationships that could be construed as a potential conflict of interest.

Publisher's Note: All claims expressed in this article are solely those of the authors and do not necessarily represent those of their affiliated organizations, or those of the publisher, the editors and the reviewers. Any product that may be evaluated in this article, or claim that may be made by its manufacturer, is not guaranteed or endorsed by the publisher.

Copyright (c) 2021 Zhu, Yang, Zeng, Li, Guan, Zhou, Wang, Liu, Gao, Qiu, Chen, Li, Zheng, Yuan, Zhang and Pan. This is an open-access article distributed under the terms of the Creative Commons Attribution License (CC BY). The use, distribution or reproduction in other forums is permitted, provided the original author(s) and the copyright owner(s) are credited and that the original publication in this journal is cited, in accordance with accepted academic practice. No use, distribution or reproduction is permitted which does not comply with these terms. 